\title{
Transcervical Radiofrequency Ablation of Symptomatic Uterine Fibroids: 2-Year Results of the SONATA Pivotal Trial
}

\author{
Charles E. Miller, $M D^{1}$ and Khadra M. Osman, $\mathrm{MD}^{2}$
}

\begin{abstract}
Objective: To report 2-year results of sonography-guided transcervical fibroid ablation (TFA) using the Sonata ${ }^{\circledR}$ system in women with symptomatic uterine fibroids.

Design: This is a prospective multicenter single-arm interventional trial.

Methods: Premenopausal women with up to 10 clinically relevant uterine fibroids, each ranging from 1 to $5 \mathrm{~cm}$ in diameter, were treated with sonography-guided TFA on an outpatient basis and returned for regular follow-up visits for 2 years. Assessed outcomes included changes in symptom severity, heath-related quality of life, general health status, work and activity limitations, treatment satisfaction, adverse events, surgical reintervention, and occurrence of pregnancy and associated outcomes.

Results: Among 147 enrolled women, 125 (85\%) returned for follow-up at 2 years. Compared with baseline, symptom severity decreased from $55 \pm 19$ to $24 \pm 18(p<0.001)$, health-related quality of life increased from $40 \pm 21$ to $83 \pm 19(p<0.001)$, and EuroQol 5-Dimension scores increased from $0.72 \pm 0.21$ to $0.89 \pm 0.14$ $(p<0.001)$. Overall treatment satisfaction at 2 years was $94 \%$. The mean percentage of missed work time, overall work impairment, and activity impairment significantly decreased at follow-up. Through 2 years, surgical reintervention for heavy menstrual bleeding was performed in $5.5 \%$ of patients. One singleton pregnancy occurred with a normal peripartum outcome.

Conclusions: TFA treatment with the Sonata system provides significant clinical improvement through 2 years postablation, with a low incidence of surgical reintervention. Other favorable outcomes included a rapid return to work and substantial improvements in quality of life, symptom severity, work productivity, and activity levels. ( J GYNECOL SURG 35:345)
\end{abstract}

Keywords: transcervical fibroid ablation, radiofrequency ablation, leiomyoma, uterine fibroids, quality of life, ultrasonography

\section{Introduction}

$\mathbf{U}$ TERINE FIBROIDS ARE a highly prevalent gynecologic condition and can be identified in at least $70 \%$ of women by the age of 50 years. ${ }^{1}$ Many women with fibroids are asymptomatic and require no intervention. However, at least one in three women with fibroids report symptoms such as heavy menstrual bleeding (HMB) and/or bulk symptoms that interfere with activities of daily living. ${ }^{2}$ Women diagnosed with uterine fibroids also have a higher risk of anemia and infertility than women without this diagnosis. ${ }^{3,4}$ Self- management of symptoms with nonprescription medication or lifestyle modification before seeking medical care is common, but often unsuccessful. ${ }^{5}$

Initial management of symptomatic fibroids may be guided by the patient's desire for future fertility. More than 200,000 hysterectomies are performed each year in the United States for the treatment of symptomatic fibroids. ${ }^{6}$ However, there is growing concern that hysterectomy for fibroid treatment is overutilized ${ }^{7}$ and patients are increasingly seeking less invasive uterus-preserving treatment options. ${ }^{5}$ Myomectomy and uterine artery embolization (UAE)

\footnotetext{
${ }^{1}$ The Advanced Gynecologic Surgical Institute, Schaumburg, Illinois.

${ }^{2}$ Fort Lauderdale Women Care, Ft. Lauderdale, Florida.

(C) Charles E. Miller and Khadra M. Osman, 2020; Published by Mary Ann Liebert, Inc. This Open Access article is distributed under the terms of the Creative Commons License (http://creativecommons.org/licenses/by/4.0), which permits unrestricted use, distribution, and reproduction in any medium, provided the original work is properly cited.
} 
are uterus-preserving alternatives to hysterectomy that may be appropriate for well-selected patients. However, the acceptability of these treatments may be limited since $79 \%$ of women with symptomatic fibroids desire treatments that do not involve invasive surgery and $65 \%$ of women younger than 40 years prefer a treatment that preserves fertility. ${ }^{5}$ In the case of UAE, future pregnancy is not recommended, and successful pregnancy outcomes are reduced after such treatment. Surgical reintervention rates for hysterectomy alternatives have been reported as high as $23.5 \%$ at 2 years. ${ }^{8-11}$ Given the lack of treatment options that align with these preferences, women with symptomatic fibroids represent an underserved population who would benefit from the development of safer, less invasive, and equally or more effective treatment options.

Use of radiofrequency (RF) ablation as a therapeutic option for solid tumors has been increasing over the past two decades among various therapeutic areas. RF ablation heats targeted tissue, causing coagulative necrosis. To better address the needs of women with symptomatic fibroids, an incisionless uterus-preserving sonography-guided transcervical fibroid ablation (TFA) outpatient procedure has been developed. In the sonography-guided transcervical ablation of uterine fibroids (SONATA) pivotal trial, performed under an investigational device exemption (IDE) from the U.S. Food and Drug Administration (FDA), clinically meaningful improvements in patient-reported symptoms, no devicerelated complications, and a surgical reintervention rate of $<1 \%$ were reported through 1 year. ${ }^{12}$ To characterize longer term safety and efficacy results with this procedure, we present 2-year results from this pivotal trial of sonographyguided transcervical RF ablation in women with symptomatic uterine fibroids.

\section{Materials and Methods}

\section{Study design}

SONATA was a prospective multicenter single-arm interventional trial of sonography-guided TFA in women with symptomatic uterine fibroids. The clinical trial was performed under an IDE approved by the FDA in the United States and the Federal Commission for Protection against Health Risks (COFEPRIS) in Mexico. Study enrollment began in April of 2015 and ended in October of 2016. Each patient provided informed consent to participate in the trial, and every clinical site obtained local institutional review board or ethics committee approval before commencing patient enrollment. The study was registered at ClinicalTrials.gov (NCT02228174).

\section{Participants}

Eligible subjects were premenopausal women aged 25 to 50 years with regular and predictable menstrual cycles, objective evidence of HMB, and with up to 10 fibroids of International Federation of Gynecology and Obstetrics (FIGO) types 1, 2, 3, 4, and/or 2-5 (transmural), each from 1 to $5 \mathrm{~cm}$ diameter. Types 5 and 6 subserous myomata were not counted in the total number of fibroids but could be ablated at the discretion of the investigator. At least one fibroid was required to have either indented or abutted the endometrial cavity (FIGO type 1, type 2, type 3, or types 2
5 fibroids). Women were excluded if they expressed a desire for future pregnancy, had any type 0 fibroids $\geq 1.0 \mathrm{~cm}$ or endometrial polyps $\geq 1.5 \mathrm{~cm}$ or multiple polyps of any size, bulk symptoms attributable to subserous fibroids, prior confounding procedures (endometrial ablation, UAE, uterine artery occlusion, or hyperthermic ablation of fibroids), uterine volume $\geq 1000 \mathrm{~cm}^{3}$, presence of tubal implants for sterilization, and/or clinically significant adenomyosis.

\section{Procedure}

Clinical sites with community or academic gynecologists with generalist experience in hysteroscopic and/or laparoscopic surgery participated in the trial. Gynecologist training for the procedure entailed didactic instruction and practice on physical uterine models with various fibroid sizes, types, and locations. The sonography-guided TFA procedure used in the trial has been described in detail elsewhere. ${ }^{12}$ The treatment device (Sonata ${ }^{\circledR}$ system; Gynesonics, Inc., Redwood City, CA) consists of an integrated intrauterine sonography probe and RF ablation handpiece that allows the gynecologist to identify, target, and ablate uterine fibroids. The integration of real-time ultrasound imaging enables the physician to visualize, target, and ablate a greater range of fibroids than could be approached through operative hysteroscopy. ${ }^{12}$ A graphical interface is displayed on the live ultrasound image that identifies the target ablation area and the extent of subablative thermal heating. The gynecologist utilizes this information to confirm the ablation is within the fibroid while confining the thermal safety border to within the uterine serosa. A single ablation may suffice to treat a fibroid, but additional ablations may be necessary depending on fibroid size, location, and geometry. Anesthesia was individualized; general anesthesia was not a requirement.

\section{Follow-up and outcomes}

Patients returned for follow-up visits at 10 days, 30 days, 3 months, 6 months, 1 year, and 2 years. Follow-up remains ongoing in this trial through 3 years. Outcomes at 2 years included changes in symptom severity, health-related quality of life (HRQL), general health, and work/activity limitations; serious adverse events; surgical reintervention for HMB; and occurrence of pregnancy and associated outcomes. Symptom severity and quality of life were assessed with the symptom severity score (SSS) and HRQL subscales of the uterine fibroid symptom and quality-of-life questionnaire. ${ }^{13}$ Scores are reported on a 0 to 100 scale where higher SSSs indicate more severe symptoms and lower HRQL scores indicate worse quality of life. Changes in general health status were assessed with the EuroQol 5Dimension (EQ-5D) questionnaire. The EQ-5D consists of five questions that provide a description of the patient's health state with scores ranging from 0 (indicating death) to 1 (indicating perfect health). Patients self-reported their perceived treatment benefit at 2 years as improved, no change, or worsened. Treatment satisfaction was measured on a 6-item scale ranging from very satisfied to very dissatisfied. The work productivity and activity impairment questionnaire: specific health problem questionnaire ${ }^{14}$ assessed change in work and activity patterns after treatment. Overall patient treatment outcome was assessed using the 
overall treatment effect (OTE) scale. Adverse events were reported according to seriousness and relationship with the device or procedure.

\section{Statistical analysis}

Safety analyses included all treated patients and efficacy analyses excluded patients who reached menopause during follow-up. Data were reported using the mean and standard deviation for normally distributed continuous outcomes, median and interquartile range for non-normally distributed continuous data, and count and frequency for categorical data. The Wilcoxon signed-rank test assessed change over time for symptom severity, HRQL, general health, and work/activity limitation outcomes. Reintervention due to HMB was analyzed using Life-Table methods, with a sensitivity analysis using binomial methods (i.e., event count divided by evaluable sample size). Data were analyzed using SAS version 9.3 (SAS Institute, Cary, NC). All statistical tests were two sided, and $p$-values of $<0.05$ indicated statistical significance.

\section{Results}

A total of 147 women (mean age 43 years, body mass index $29 \mathrm{~kg} / \mathrm{m}^{2}$ ) were enrolled at 22 sites (21 in the United States, 1 in Mexico). Demographic characteristics of the patients are provided in Table 1. All patients presented with HMB and their general health status measured on the EQ$5 \mathrm{D}$ was below the 25 th percentile compared with sex- and age-matched population norms. ${ }^{15} \mathrm{~A}$ mean of $3.0( \pm 2.1)$ fibroids per patient were treated with transcervical RF ablation. Patient characteristics and procedural details have been previously reported. ${ }^{12} \mathrm{~A}$ total of $125(85 \%)$ patients returned for follow-up at 2 years. Six patients missed the 2-year follow-up visit and 16 patients withdrew from the study before the 2-year follow-up visit (none due to an adverse event).

Over the 2-year follow-up period, mean values on the SSS decreased from $55 \pm 19$ to $24 \pm 18(p<0.001)$, HRQL scores increased from $40 \pm 21$ to $83 \pm 19(p<0.001)$ (Fig. 1), and EQ-5D scores increased from $0.72 \pm 0.21$ to $0.89 \pm 0.14$ $(p<0.001)$ (Fig. 2). Patient satisfaction with treatment at 2

Table 1. Baseline Patient Characteristics

\begin{tabular}{lc}
\hline Variable & Value $(\mathrm{n}=147)$ \\
\hline Age, years & $42.9 \pm 4.3[31,50]$ \\
Ethnicity & $43(29.3)$ \\
$\quad$ Hispanic or Latina & $104(70.7)$ \\
Not Hispanic or Latina & \\
Race & $3(2.0)$ \\
American Indian or Alaska Native & $2(1.4)$ \\
Asian & $49(33.3)$ \\
Black or African American & $1(0.7)$ \\
Native Hawaiian or other Pacific & $60(40.8)$ \\
White & $33(22.4)$ \\
Other & \\
\hline
\end{tabular}

Values are mean \pm standard deviation [minimum, maximum], or count (percentage).

${ }^{\mathrm{a}}$ Patients can be counted more than once if multiple races were indicated.

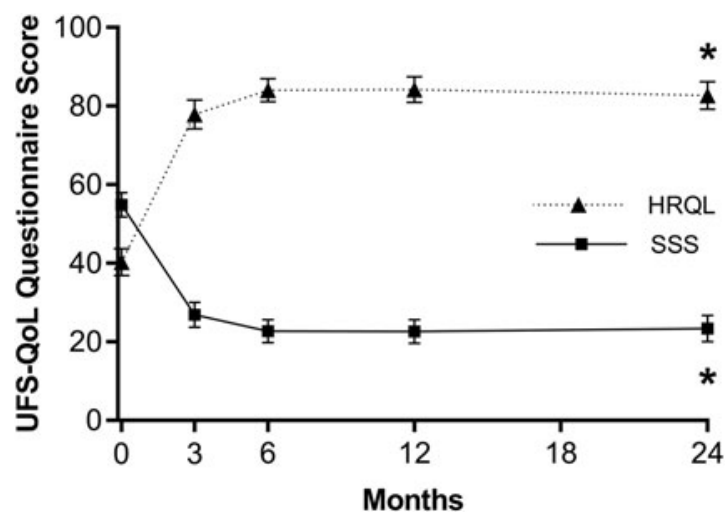

FIG. 1. Change in SSS and HRQL subscales of the UFSQoL questionnaire for 2 years after sonography-guided transcervical fibroid ablation. Lower SSSs indicate less severe symptoms. Higher HRQL scores indicate better quality of life. Plotted values are mean and $95 \%$ confidence interval. ${ }^{*} p<0.001$ for change from baseline. HRQL, health-related quality of life; SSS, symptom severity score; UFS-QoL, uterine fibroid symptom and quality of life.

years was $94 \%$ ( $75 \%$ of patients reported they were very satisfied, $13 \%$ were moderately satisfied, $6 \%$ were somewhat satisfied, $0 \%$ were somewhat dissatisfied, $4 \%$ were moderately dissatisfied, and $2 \%$ were very dissatisfied). At 2 years, $88 \%$ of patients reported improvement in fibroid symptoms on the OTE questionnaire compared with baseline. Indicators of work impairment due to fibroid symptoms significantly improved from baseline to 2 years. The percentage of missed work time significantly decreased from a mean of $2.9 \%$ to $1.3 \%(p<0.001)$ and the overall percentage of work impairment also demonstrated significant improvement, being reduced from a mean of $51 \%$ to $14 \%(p<0.001)$. Patients also reported significant reductions in the percentage of activity impairment due to fibroid symptoms over this period (mean of $58 \%$ to $14 \%$, $p<0.001)$.

One-year safety outcomes with sonography-guided transcervical RF ablation in this trial were previously reported. ${ }^{12}$ In brief, procedure-related serious adverse events

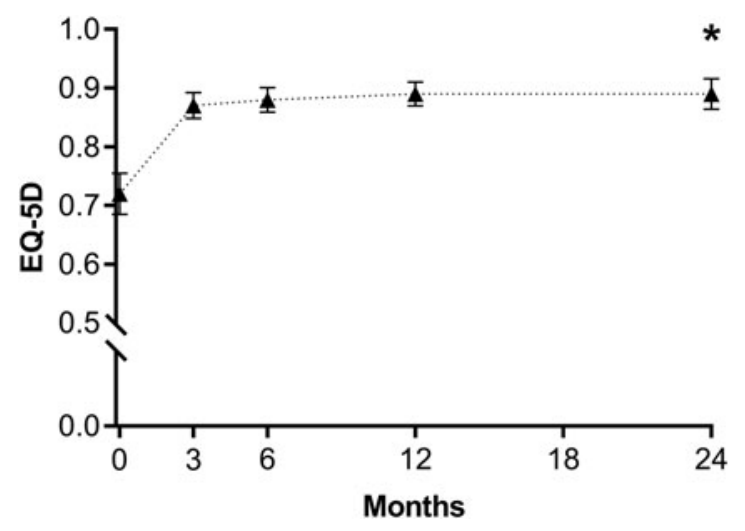

FIG. 2. Change in EQ-5D questionnaire score for 2 years after sonography-guided transcervical fibroid ablation. Plotted values are mean and $95 \%$ confidence interval. ${ }^{*} p<0.001$ for change from baseline. EQ-5D, EuroQol 5-Dimension. 
were reported in $2(1.4 \%)$ patients during the first year of follow-up without any device-related adverse events during this period. Between the 1- and 2-year follow-up visits, there were no serious adverse events and no adverse events related to the device or procedure. The cumulative rate of surgical reintervention for HMB through 2 years was $5.2 \%$ (95\% confidence interval [CI]: 2.5\%-10.6\%) using LifeTable methods and 5.5\% (95\% CI: 2.2\%-11.0\%) using binomial methods. One singleton pregnancy was reported in a 36-year-old multigravida who conceived 22 months after ablation. The patient delivered a liveborn male infant at $382 / 7$ weeks gestation by elective repeat cesarean section with Apgar scores of $9^{1} / 10^{5}$ and a birth weight of $4005 \mathrm{~g}$. Visual inspection of the endometrial cavity appeared within normal limits and there was no evidence of uterine dehiscence or rupture.

\section{Discussion}

Women with symptomatic uterine fibroids are often treated with hysterectomy and other significantly invasive and potentially morbid procedures. Although hysterectomy is definitive treatment for fibroids, it removes the possibility of future pregnancy, and there are concerns about potential need for blood products; complications (including long-term effects such as pelvic prolapse), lost work time; possible earlier menopause; and increased osteoporosis risk. $5,16,17$

Most affected women prefer uterine-conserving treatments irrespective of their desire for childbearing. ${ }^{5}$ Therefore, a safe, effective, and convenient less invasive treatment option for symptomatic fibroids would be welcomed by patients and health care providers alike. Sonographyguided TFA was developed to bridge this therapeutic gap. Although promising results with this technology have been reported in previous studies, ${ }^{18,19}$ this study is the largest conducted to date. The 2-year results of the SONATA Pivotal IDE Trial demonstrate that sonographyguided TFA is a safe outpatient incisionless and uteruspreserving procedure, which provides significant durable symptom relief, and significantly improves general and condition-specific quality of life through 2 years.

Results with sonography-guided TFA in the current trial are comparable with those reported in previously published studies with this technology. A clinical trial (Fibroid Ablation Study-EU) in Europe and Mexico that followed 50 patients treated with the Sonata System (previously VizAblate ${ }^{\circledR}$ ) for 1-year reported encouraging results. ${ }^{19}$ During follow-up, fibroid volume decreased by $67 \%$, fibroid symptoms significantly improved, and overall safety was excellent. Long-term results from the VITALITY Clinical Study demonstrated that symptom resolution persisted in 17 women treated with the Sonata System for 5.4 years mean follow-up, with no surgical reintervention for the first 3.5 years, a $11.8 \%$ rate of surgical reintervention at 5 years for $\mathrm{HMB}$, and an overall surgical reintervention event rate of $2.2 \%$ per year. $^{20}$ One-year results from the current SONATA trial were previously reported in which fibroid volume decreased by $62 \%$, fibroid symptoms significantly improved, and the surgical reintervention rate was $0.7 \%$. The current report extends these previous results to 2 years, in which efficacy was durably maintained, one pregnancy with a normal peripartum outcome resulted, and no new safety concerns were identified. The 2-year surgical reintervention rate reported herein for TFA of uterine fibroids was $5.5 \%$. This is noteworthy, considering the rates of reintervention for other procedures reported at 2 years were $23.5 \%$ for UAE, $18 \%$ for hysteroscopic myomectomy, $19 \%$ for endometrial ablation, and $8 \%$ for laparoscopic myomectomy. ${ }^{9,21}$

TFA was performed with acceptable safety in this study. Transcervical access involves no incisions and avoids the peritoneal cavity, which minimizes iatrogenic complication risks inherent with transperitoneal surgery such as wound infection or ureteral injury. Patients were discharged $\sim 2$ hours after the procedure and treatment satisfaction was high. Although general anesthesia was used in $50 \%$ of patients largely due to patient or anesthesiologist preference, it is not a requirement and most patients treated with conscious sedation experienced minimal procedural discomfort. Because a varied mixture of community and academic generalist gynecologists were recruited and provided treatment after a brief standardized didactic and practical training program, this procedure appears to be generalizable to a broad range of gynecologists since specialized sonography expertise was neither required nor assumed. As with any new treatment, thoughtful patient selection and careful attention to the prescribed procedural technique are important for optimal results. Thorough differential diagnosis should be performed to determine whether patient symptoms are attributable to uterine fibroids or other causes such as anovulation, adenomyosis, or bleeding disorders. Shared decision-making between patient and provider should dictate the preferred procedure for symptomatic fibroid treatment. Published clinical results demonstrate that TFA is a safe and effective option that can be included in the gynecologist's armamentarium of treatment options among patients seeking treatment for symptomatic fibroids.

\section{Conclusion}

TFA treatment with the Sonata system provides significant clinical improvement through 2 years postablation, with a low incidence of surgical reintervention and a favorable safety profile. Other outcomes included a rapid return to work and substantial improvements in quality of life, symptom severity, work productivity, and activity levels.

\section{Acknowledgments}

The authors thank David B. Toub, MD, MBA, Taraneh G. Farazi, $\mathrm{PhD}$, and Larry Miller, $\mathrm{PhD}$, for editorial assistance, and QST Consultations, LTD for statistical support.

\section{Author Disclosure Statement}

The institutions of the authors received research support from Gynesonics, Inc. for participation in the SONATA clinical trial. The authors have received travel and lodging expenses for attendance at investigator meetings. C.M. is a consultant to Gynesonics.

\section{Funding Information}

Gynesonics. Inc. provided funding for this study. 


\section{References}

1. Baird DD, Dunson DB, Hill MC, Cousins D, Schectman JM. High cumulative incidence of uterine leiomyoma in black and white women: Ultrasound evidence. Am J Obstet Gynecol 2003;188:100.

2. Zimmermann A, Bernuit D, Gerlinger C, Schaefers M, Geppert K. Prevalence, symptoms and management of uterine fibroids: An international internet-based survey of 21,746 women. BMC Womens Health 2012;12:6.

3. Bartels CB, Cayton KC, Chuong FS, Holthouser K, Arian SE, Abraham T, Segars JH. An evidence-based approach to the medical management of fibroids: A systematic review. Clin Obstet Gynecol 2016;59:30.

4. Levy G, Hill MJ, Beall S, Zarek SM, Segars JH, Catherino WH. Leiomyoma: Genetics, assisted reproduction, pregnancy and therapeutic advances. J Assist Reprod Genet 2012;29:703.

5. Borah BJ, Nicholson WK, Bradley L, Stewart EA. The impact of uterine leiomyomas: A national survey of affected women. Am J Obstet Gynecol 2013;209:319.e1.

6. National Institutes of Health. Uterine Fibroids. 2018. Online document at: https://report.nih.gov/nihfactsheets/viewfact sheet.aspx?csid=50 Accessed January 10, 2019.

7. Corona LE, Swenson CW, Sheetz KH, et al. Use of other treatments before hysterectomy for benign conditions in a statewide hospital collaborative. Am J Obstet Gynecol 2015;212:304.e1.

8. Ananthakrishnan G, Murray L, Ritchie M, et al. Randomized comparison of uterine artery embolization (UAE) with surgical treatment in patients with symptomatic uterine fibroids (REST trial): Subanalysis of 5-year MRI findings. Cardiovasc Intervent Radiol 2013;36:676.

9. Davis MR, Soliman AM, Castelli-Haley J, Snabes MC, Surrey ES. Reintervention rates after myomectomy, endometrial ablation, and uterine artery embolization for patients with uterine fibroids. J Womens Health (Larchmt) 2018;27:1204.

10. Funaki K, Fukunishi H, Sawada K. Clinical outcomes of magnetic resonance-guided focused ultrasound surgery for uterine myomas: 24-month follow-up. Ultrasound Obstet Gynecol 2009;34:584.

11. van der Kooij SM, Hehenkamp WJ, Volkers NA, Birnie E, Ankum WM, Reekers JA. Uterine artery embolization vs hysterectomy in the treatment of symptomatic uterine fi- broids: 5-year outcome from the randomized EMMY trial. Am J Obstet Gynecol 2010;203:105.e1.

12. Chudnoff S, Guido R, Roy K, Levine D, Mihalov L, GarzaLeal JG. Ultrasound-guided transcervical ablation of uterine leiomyomas. Obstetr Gynecol 2019;133:13.

13. Reilly MC, Zbrozek AS, Dukes EM. The validity and reproducibility of a work productivity and activity impairment instrument. Pharmacoeconomics 1993;4:353.

14. Harding G, Coyne KS, Thompson CL, Spies JB. The responsiveness of the uterine fibroid symptom and healthrelated quality of life questionnaire (UFS-QOL). Health Qual Life Outcomes 2008;6:99.

15. Szende A, Janssen B, Cabases J. Self-Reported Population Health: An International Perspective Based on EQ-5D. New York: Springer Open, 2014.

16. Farquhar CM, Sadler L, Harvey SA, Stewart AW. The association of hysterectomy and menopause: A prospective cohort study. BJOG 2005;112:956.

17. Robinson D, Thiagamoorthy G, Cardozo L. Posthysterectomy vaginal vault prolapse. Maturitas 2018;107:39.

18. Bongers M, Brolmann H, Gupta J, Garza-Leal JG, Toub D. Transcervical, intrauterine ultrasound-guided radiofrequency ablation of uterine fibroids with the VizAblate(R) System: Three- and six-month endpoint results from the FAST-EU study. Gynecol Surg 2015;12:61.

19. Brolmann H, Bongers M, Garza-Leal JG, Gupta J, Veersema S, Quartero R, Toub D. The FAST-EU trial: 12Month clinical outcomes of women after intrauterine sonography-guided transcervical radiofrequency ablation of uterine fibroids. Gynecol Surg 2016;13:27.

20. Garza-Leal JG. Long-term clinical outcomes of transcervical radiofrequency ablation of uterine fibroids: The VITALITY study. J Gynecol Surg. 2018;35:19.

21. van der Kooij SM, Bipat S, Hehenkamp WJ, Ankum WM, Reekers JA. Uterine artery embolization versus surgery in the treatment of symptomatic fibroids: A systematic review and metaanalysis. Am J Obstet Gynecol 2011;205:317.e1.

Address correspondence to: Charles E. Miller, MD The Advanced Gynecologic Surgical Institute 120 Osler Drive, Suite 100 Naperville IL 60540

E-mail: chuckmillermd@gmail.com 